\title{
Short- And Long-Term Effects of Breast Cancer Diagnosis and Adjuvant Endocrine Treatment on Life Satisfaction
}

\author{
Anna Skördåker ${ }^{1}$, Anna von Wachenfeldt ${ }^{2}$, Elisabet Lidbrink ${ }^{3}$, Johanna Stjärnfeldt ${ }^{4}$, Aina \\ Johnsson ${ }^{5}$ \\ ${ }^{1}$ Rehabcenter Sfären, Box 7023, SE-170 07 Solna, Sweden \\ ${ }^{2}$ Department of Oncology, Karolinska University Hospital, Södersjukhuset, SE- 11883 Stockholm, Sweden \\ ${ }^{3}$ KarolinskaInstitutet Department of Oncology and Pathology, SE-171 76 Stockholm, Sweden \\ ${ }^{4}$ KarolinskaInstitutet, Department of Neurobiology, Care Sciences and Society, Division of Social Work, SE-14186 \\ Huddinge, Sweden \\ ${ }^{5}$ Karolinska University Hospital, Department of Social Work, SE-141 86, Stockholm, Sweden
}

\begin{abstract}
:
Purpose: The aim of this study was to examine if breast cancer patients' estimated life satisfaction was affected during adjuvant endocrine treatment, if life satisfaction changed over time and if there were any differences in life satisfaction between breast cancer patients who experienced side effects from the treatment and those who did not.
\end{abstract}

Material and methods: Consecutive breast cancer patients $(n=68)$ referred for adjuvant endocrine treatment after radical breast cancer surgery at an oncology clinic were enrolled from November 2002 to August 2004. The patients scored their life satisfaction at inclusion, during treatment, and about 2 years after completing treatment. The questionnaire LiSat-11 was used. A report of side effects in medical records was also carried out. Comparisons, focusing on life satisfaction, between patients with and without side effects were made.

Results: Of the patients in the study group $42(61.8 \%)$ had musculoskeletal symptoms noted in their medical records, $48(70.6 \%)$ reported menopausal symptoms and $40(58.5 \%)$ had psychological symptoms. At inclusion and during the treatment the study group estimated lower life satisfaction in most domains compared with a reference group. At post-treatment the study group still estimated lower life satisfaction in the domains sexual life, partner relationship, somatic and psychological health compared with a reference group. The study group estimated lower life satisfaction at post-treatment compared to inclusion in the domains sexual life and partner relationship. In the other domains an improvement was observed when life satisfaction at post-treatment was compared with inclusion. Patients with side effects estimated lower life satisfaction in most domains compared with those that experienced no side effects

Conclusion: The important findings are that the studied breast cancer patients receiving endocrine treatment estimated their life satisfaction lower in most domains compared with the normal population already at inclusion. During the treatment, life satisfaction further deteriorated but the patients had recovered in most domains post-treatment. Notable is the deterioration in sexual life and partner relationship at post-treatment compared with inclusion. This study indicates that long-term effects of endocrine breast cancer therapy need to be further researched, with a particular focus on issues relating to sexuality and relationships.

Key words: breast cancer; adjuvant therapy; endocrine therapy; side effects; life satisfaction

\section{INTRODUCTION}

Breast cancer constitutes $30 \%$ of female cancer. In Sweden 8,490 patients were diagnosed with the disease during 2012. The median age for diagnosis is approximately 64 years of age ${ }^{1}$. The incidence of breast cancer has increased over the last decades, but at the same time the mortality rate has declined and today the 5-year (cumulative) relative survival ratio is nearly $90 \%{ }^{1}$. Breast cancer is, in more than $70 \%$ of cases, hormone-receptor positive, i.e. it is stimulated to grow by the woman's own sex hormones. Therefore, patients that have undergone breast cancer surgery and have a hormone-receptor positive tumour are almost always offered adjuvant endocrine treatment for at least 5 years in order to reduce potentially remaining tumour cells. Treatment with tamoxifen for five years reduces

${ }^{1}$ Corresponding Author: aina.johnsson@karolinska.se 
the recurrence rate by $39 \%$ over the first 10 years $^{2}$ and if aromatase inhibitor (AI) is included in the treatment the risk is reduced even more ${ }^{3}$. If breast cancer treatment is to be successful, adherence to treatment is important. A Scottish study showed a significantly lower survival rate among breast cancer patients who were non-adherent to offered tamoxifen treatment ${ }^{4}$. Not all patients adhere to the adjuvant endocrine treatment, various studies report on very low adherence rates after one year or more. In a Swedish study the level of adherence after three years was $69 \%$.

Endocrine treatment with tamoxifen and AI causes a risk of several side effects. The side effects from tamoxifen are mainly menopausal symptoms such as hot flushes, sweating, gynaecological symptoms and fatigue ${ }^{6}$. Those symptoms can result in worsening quality of life due to pain and sexual dysfunction ${ }^{7}$. Up to $46 \%$ of the patients treated with AI will develop one or more of the symptoms osteoarthritis, bursitis, carpal tunnel syndrome and tendonitis ${ }^{8}$. A patient with pre-existing mild- to moderate joint symptoms/arthralgia syndrome faces a risk of a significant exacerbation of the symptoms. AI-induced/-associated arthralgia during the endocrine treatment can dramatically alter the patient's normal daily functioning ${ }^{9-11}$. A study by Huang and colleagues stated that fatigue is a major problem in most breast cancer patients during the adjuvant endocrine treatment ${ }^{12}$. Other studies have shown that breast cancer patients are at high risk of developing depression, particularly in a postmenopausal or oestrogen deficiency state ${ }^{13}$.

There are a large number of papers that highlight the health-related quality of life of patients diagnosed with breast cancer disease, but far fewer highlight the quality of life/life satisfaction in patients receiving adjuvant endocrine treatment. There is no study that describes how life satisfaction varies over time from baseline through treatment and up to two years post-treatment.

The aim of this study was to examine whether the included breast cancer patients' estimated life satisfaction was affected during the endocrine treatment, and whether this changed over time, and if there was any difference in life satisfaction between patients who experienced side effects and patients who did not.

\section{MATERIAL AND METHODS}

\subsection{Study Design}

The study is based on questionnaires used on a prospective cohort of 68 breast cancer patients and data from their medical records. In this study the concept 'life satisfaction' is used. The definition of 'satisfaction with life as a whole' is to which extent a person is able to achieve vital goals ${ }^{14}$.

\subsection{Sample and Settings}

The study group included patients who had undergone surgery for early stage breast cancer. After surgery the patients had been prescribed adjuvant endocrine treatment at the Department of Oncology, Karolinska University Hospital, Södersjukhuset in Stockholm. Patients were approached consecutively from November 2002 to January 2003 and from November 2003 to August 2004 and invited to participate in the study, around 6 weeks after surgery. All patients were followed for 5 years. The study was completed after 5 years and only those who had completed the treatment remained in the study. Other inclusion criteria were: breast cancer patients between the ages of 18 and 64 years with a primary invasive, surgically and radically treated breast cancer, without clinical evidence of distant metastases. Patients had to live in Stockholm County, be working at least half-time at the time of diagnosis and be able to read and understand Swedish. Exclusion criteria were: inoperable breast cancer or other serious disease, recurrence or suspicions of recurrence of breast cancer disease, or sick leave for more than 6 months at the time of diagnosis. In total 68 patients met the criteria for inclusion in the study.

This study has its origin in another study with the purpose of examining how breast cancer patients' lives change in the years following diagnosis, with a focus on return to work (Johnsson et al., 2011). Of the 102 patients enrolled in that study, 68 patients met the inclusion criteria for this study. The reasons for 34 patients being excluded were: 14 patients either did not receive endocrine treatment or refused the treatment, nine patients had died, five patients had recurrence of their breast cancer disease, five had moved from the Stockholm area and one patient had another serious disease. Of the 68 who met the inclusion criteria 63 completed the questionnaire at inclusion.

\subsection{Data Assessment - Procedure}

The patients scored their life satisfaction at inclusion in the study, after about 12 months following inclusion (during treatment) and between one-and-a-half and up to two years after completed endocrine treatment (post-treatment). To measure life satisfaction the generic self-report questionnaire LiSat-11 was used. LiSat-11 is a non-diagnosis specific questionnaire developed by Fugl-Meyer to measure life satisfaction (Fugl-Meyer et al. 2002). Li-Sat 11 covers 
life satisfaction in 11 life domains: life in general, vocational situation, financial situation, leisure situation, contact with friends, sexual life, activities of daily life, family life, partner relationship, somatic health and psychological health. For each item, the patients selected a level of satisfaction on a six-grade scale: very dissatisfied (1), dissatisfied (2), rather dissatisfied (3), rather satisfied (4), satisfied (5) and very satisfied (6). For each item scores were dissatisfied (scores 1-4) or satisfied (scores grad 5-6). The instrument has reference values for a normal population, aged 18-64, which is based on data comprising 2,533 people (1,207 patients) of working age in Sweden (FuglMeyer et al. 2002).

The medical record review covered five years of medical records from patients' scheduled appointments at the Department of Oncology. The review covered appointments at 1, 6, 12, 24, 36 and 60 months after the start of the endocrine treatment. A template was designed in which the data was collected and classified. In this template musculoskeletal disorders were categorised as: joint stiffness, joint pain, carpal tunnel syndrome and unspecified pain. Menopausal symptoms also included sexual dysfunction. Psychological symptoms were categorised as: anxiety, depression, irritability, cognitive dysfunction, fatigue, and sleeping problems. The medical record review also provided data on treatment characteristics.

\subsection{Statistical Methods}

Percentage, mean, and median values are presented for the descriptions (characteristics, life satisfaction, and symptoms). In order to compare whether the differences in estimated life satisfaction were significant between measurements, the $t$-test for paired comparisons was used. The measurements paired and compared were: inclusion occasion compared with during treatment occasion, inclusion occasion compared with post-treatment occasion, and during treatment occasion compared with post-treatment occasion. The results from the $t$-test were compared with a nonparametric alternative, Wilcoxon sign rank test. Since both tests gave similar results, deviation from a normal distribution was not a problem. The interpretations of the results could therefore be based on the $p$-values from the $t$-test. Chosen level of significance test was $p<0.05$ and all the tests were two-tailed. At the statistical analysis SPSS TM version 21 was used. Comparisons were made between patients with and without symptoms, as regards life satisfaction using the Mann Whitney $\mathrm{U}$ test. Chosen level of significance test in the study was $\mathrm{p}<0.05$.

\subsection{Ethics}

The study was approved by the Research Ethics Committee's South, supplemented with an additional application to the Regional Ethical Review Board in Stockholm. Written informed consent was obtained from all patients before inclusion in the study. The patients received oral and written information about the study, stressing voluntary participation and possible withdrawal at any time. Thereafter, they were given the first questionnaire and a prepaid return envelope. The study is adherent to the ethical principles stipulated in The Helsinki Declaration (2013).

\section{RESULTS}

The demographic and clinical characteristics are presented in Table I. The median age in the study group was 53 years of age at inclusion and 60 years of age at the end of the study. A majority, 57 out of 68 patients, were born in Sweden. The most common endocrine treatment was tamoxifen either alone or in combination with goserelin. Approximately half of the patients had been prescribed such treatment. A nearly equal number of patients had been prescribed AI. One woman had been prescribed goserelin solely. In total 42 of the studied patients had musculoskeletal symptoms noted in their medical records during the treatment period, which represents $61.8 \%$ of the study group. The number of patients with menopausal symptoms was 48 (70.6\%) and with psychological symptoms 40 $(58.5 \%)$.

Table I. Demographic and clinical characteristics among 68 women offered endocrine therapy as adjuvant treatment

\begin{tabular}{|l|c|}
\hline Characteristics at inclusion & $\mathrm{n}(68)$ \\
\hline Age mean (range) & $53(35-62)$ \\
\hline Marital status & \\
\hline Single & 22 \\
\hline Living with partner & 46 \\
\hline Biological children & 13 \\
\hline No biological children & 55 \\
\hline$\geq 1$ biological child & \\
\hline
\end{tabular}


American Research Journal of Nursing, Volume 1, Issue 3, 2015

ISSN 2379-2922

\begin{tabular}{|l|c|}
\hline Birth Sweden & 57 \\
\hline Education & 19 \\
\hline Nine year school & 24 \\
\hline Upper secondary school & 25 \\
\hline College & 16 \\
\hline Work & 51 \\
\hline Manual labour & 1 \\
\hline Not manual labour & 27 \\
\hline Missing data & 40 \\
\hline Surgery & 1 \\
\hline Ablatio & 56 \\
\hline Sector resection & 19 \\
\hline No surgery & 24 \\
\hline Axillary node dissection & 36 \\
\hline Sentinel node biopsy & 10 \\
\hline Chemotherapy & \\
\hline Radiotherapy (RT) & 31 \\
\hline RT only to breast parenchyma & 1 \\
\hline RT to breast/chest wall and regional nodes & 28 \\
\hline Prescribed endocrine therapy & 8 \\
\hline Aromatase inhibitor & \\
\hline Aromatase inhibitor + goserelin & \\
\hline Tamoxifen & \\
\hline Tamoxifen + goserelin & \\
\hline
\end{tabular}

Table II describes the estimated life satisfaction during the study period compared to values for a normal population, aged 18-64 (the reference group). The results at the different measurements were: at inclusion the study group estimated lower life satisfaction in all domains except vocational situation, financial situation and contact with friends. During treatment the study group estimated lower life satisfaction in all domains except vocational situation and financial situation. At post-treatment the study group still estimated lower life satisfaction in the domains sexual life, activities of daily life, partner relationship, somatic health and psychological health compared to the reference group.

Table II. Life satisfaction at inclusion $(n=68)$, during treatment $(n=63)$ and post-treatment $(n=52)$ compared with the reference group.

\begin{tabular}{|l|l|l|l|l|l|l|l|}
\hline Life satisfaction & \multicolumn{2}{l|}{$\begin{array}{l}\text { Study group at in- } \\
\text { clusion }\end{array}$} & \multicolumn{2}{l|}{$\begin{array}{l}\text { Study group during } \\
\text { therapy }\end{array}$} & \multicolumn{2}{l|}{$\begin{array}{l}\text { Study group post- } \\
\text { treatment }\end{array}$} & Reference group \\
\hline $\begin{array}{l}\text { Satisfied/very } \\
\text { satisfied }\end{array}$ & $\mathrm{f} / \mathrm{n}$ & $\%$ & $\mathrm{f} / \mathrm{n}$ & $\%$ & $\mathrm{f} / \mathrm{n}$ & $\%$ & $\%$ \\
\hline Life as a whole & $40 / 68$ & 59 & $33 / 63$ & 52 & $38 / 52$ & 73 & 70 \\
\hline $\begin{array}{l}\text { Vocational } \\
\text { situation }\end{array}$ & $40 / 68$ & 59 & $34 / 62$ & 55 & $30 / 47$ & 64 & 54 \\
\hline $\begin{array}{l}\text { Financial situa- } \\
\text { tion }\end{array}$ & $34 / 68$ & 50 & $33 / 63$ & 52 & $35 / 52$ & 67 & 39 \\
\hline $\begin{array}{l}\text { Leisure situa- } \\
\text { tion }\end{array}$ & $35 / 67$ & 52 & $34 / 63$ & 48 & $40 / 51$ & 78 & 57 \\
\hline $\begin{array}{l}\text { Contact with } \\
\text { friends }\end{array}$ & $49 / 68$ & 72 & $42 / 62$ & 68 & $38 / 52$ & 73 & 69 \\
\hline Sexual life & $30 / 66$ & 46 & $20 / 60$ & 33 & $11 / 46$ & 24 & 56 \\
\hline $\begin{array}{l}\text { Activities of } \\
\text { daily life }\end{array}$ & $62 / 68$ & 91 & $55 / 63$ & 87 & $50 / 52$ & 96 & 97 \\
\hline Family life & $50 / 66$ & 76 & $41 / 60$ & 68 & $39 / 45$ & 87 & 81 \\
\hline $\begin{array}{l}\text { Partner rela- } \\
\text { tionship }\end{array}$ & $42 / 67$ & 63 & $42 / 62$ & 68 & $29 / 39$ & 74 & 82 \\
\hline Somatic health & $33 / 68$ & 49 & $30 / 63$ & 48 & $33 / 52$ & 64 & 77 \\
\hline
\end{tabular}


American Research Journal of Nursing, Volume 1, Issue 3, 2015

ISSN 2379-2922

\begin{tabular}{|l|l|l|l|l|l|l|l|}
\hline $\begin{array}{l}\text { Psychological } \\
\text { health }\end{array}$ & $42 / 68$ & 62 & $33 / 63$ & 52 & $38 / 52$ & 73 & 81 \\
\hline
\end{tabular}

During treatment the study group estimated lower life satisfaction compared with the measurement at inclusion in almost all domains, but the result was only significant within the domain sexual life (Table III).

There was an improvement in all domains except sexual life (significant) and partner relationship in how the study group estimated life satisfaction at measurement at post-treatment compared to inclusion. In the domains vocational situation, financial situation, leisure situation, contact with friends and psychological health the improvement was significant (Table IV).

Table III. Life satisfaction in the study group at inclusion and during treatment

\begin{tabular}{|l|l|l|l|l|}
\hline Life satisfaction & $\mathbf{n}$ & Mean at inclusion & Mean during therapy & p-value \\
\hline Life as a whole & 62 & 4.73 & 4.42 & 0,110 \\
\hline Vocational situation & 62 & 4.47 & 4,35 & 0.482 \\
\hline Financial situation & 63 & 4,30 & 4,17 & 0,329 \\
\hline Leisure situation & 62 & 4,42 & 4,34 & 0.439 \\
\hline Contact with friends & 62 & 4,97 & 4,87 & 0,370 \\
\hline Sexual life & 60 & 3,90 & 3,50 & 0.010 \\
\hline Activities of daily life & 63 & 5,63 & 5.59 & 0,605 \\
\hline Family life & 60 & 4,98 & 4,98 & 1.000 \\
\hline Partner relationship & 61 & 4,67 & 4,69 & 0,912 \\
\hline Somatic health & 63 & 4,40 & 4,22 & 0.094 \\
\hline Psychological health & 63 & 4,54 & 4,41 & 0.350 \\
\hline
\end{tabular}

T-test for paired comparisons was used in the analysis

Table IV. Life satisfaction in the study group at inclusion and post-treatment

\begin{tabular}{|l|l|l|l|l|}
\hline Life satisfaction & $\mathbf{n}$ & Mean at inclusion & Mean post-treatment & p-value \\
\hline Life as a whole & 52 & 4,67 & 4.98 & 0,400 \\
\hline Vocational situation & 47 & 4,47 & 4.77 & 0,019 \\
\hline Financial situation & 52 & 4.46 & 4,79 & 0,046 \\
\hline Leisure situation & 51 & 4,47 & 4,88 & 0.000 \\
\hline Contact with friends & 52 & 5,08 & 5,12 & 0.007 \\
\hline Sexual life & 46 & 4,09 & 3,18 & 0.000 \\
\hline Activities of daily life & 52 & 5,71 & 5,79 & 0.192 \\
\hline Family life & 45 & 5,16 & 5,20 & 0.796 \\
\hline Partner relationship & 39 & 5,13 & 4,95 & 0.420 \\
\hline Somatic health & 52 & 4,31 & 4,58 & 0.090 \\
\hline Psychological health & 52 & 4,56 & 4,92 & 0.007 \\
\hline
\end{tabular}

T-test for paired comparisons was used in the analysis

There was an improvement in all domains - except sexual life - in how the study group estimated life satisfaction at measurement at post-treatment compared to during treatment. In the domains life as a whole, financial situation, leisure situation, contact with friends, somatic health and psychological health the result was significant. Within the domain sexual life there was deterioration (Table V).

Table V: Life satisfaction in the study group during therapy and post treatment

\begin{tabular}{|l|l|l|l|l|}
\hline Life satisfaction & $\mathbf{n}$ & Mean during therapy & Mean post-treatment & p-value \\
\hline Life as a whole & 49 & 4.53 & 5.02 & 0.001 \\
\hline Vocational situation & 45 & 4,38 & 4.78 & 0.063 \\
\hline Financial situation & 49 & 4,27 & 4,78 & 0,012 \\
\hline Leisure situation & 48 & 4,38 & 4.94 & 0,001 \\
\hline Contact with friends & 48 & 4.88 & 5.19 & 0.038 \\
\hline
\end{tabular}


American Research Journal of Nursing, Volume 1, Issue 3, 2015

ISSN 2379-2922

\begin{tabular}{|l|l|l|l|l|}
\hline Sexual life & 43 & 3,63 & 3,27 & 0,117 \\
\hline Activities of daily life & 49 & 5,65 & 5,80 & 0.197 \\
\hline Family life & 42 & 5.10 & 5,29 & 0,282 \\
\hline Partner relationship & 36 & 5,00 & 5,06 & 0,822 \\
\hline Somatic health & 49 & 4.22 & 4,63 & 0.017 \\
\hline Psychological health & 49 & 4.51 & 4.98 & 0.009 \\
\hline
\end{tabular}

T-test for paired comparisons was used in the analysis.

Patients with reported symptoms during the endocrine treatment, especially psychological symptoms, had in general lower estimated life satisfaction than patients without symptoms. Patients with psychological symptoms reported lower life satisfaction in the domain life as a whole, financial situation, leisure situation, contact with friends and psychological health. Also menopausal symptoms affected the estimated life satisfaction - especially the vocational situation and the psychological health. Reported musculoskeletal symptoms seem to have affected the estimated life satisfaction to a lesser extent (Table VI).

Table VI. Self-rated life satisfaction during treatment by symptoms group.

\begin{tabular}{|c|c|c|c|c|c|c|c|c|c|c|c|}
\hline $\begin{array}{l}\text { Musculoskelet } \\
\text { al symptoms }\end{array}$ & $\mathrm{n}$ & $\begin{array}{l}\text { Median } \\
\text { rank } \\
\text { rank }\end{array}$ & p-values & $\begin{array}{l}\text { Menopausal } \\
\text { symptoms }\end{array}$ & $\mathrm{n}$ & $\begin{array}{l}\text { Median } \\
\text { rank } \\
\text { Rank }\end{array}$ & $\begin{array}{l}\mathrm{p} \text { - } \\
\text { values }\end{array}$ & $\begin{array}{l}\text { Psychological } \\
\text { symptoms } \\
\text { symptomsdoso } \\
\text { rder } \\
\text { besvär }\end{array}$ & $\mathrm{n}$ & $\begin{array}{l}\text { Median } \\
\text { rank }\end{array}$ & -values \\
\hline $\begin{array}{l}\text { Life as a } \\
\text { whole }\end{array}$ & & & & $\begin{array}{l}\text { Life as a } \\
\text { whole }\end{array}$ & & & & $\begin{array}{l}\text { Life as a } \\
\text { whole }\end{array}$ & & & \\
\hline $\begin{array}{l}\text { Yes } \\
\text { No } \\
\text { Total }\end{array}$ & $\begin{array}{l}39 \\
24 \\
63\end{array}$ & $\begin{array}{l}31,45 \\
32,90\end{array}$ & 0,752 & $\begin{array}{l}\text { Yes } \\
\text { No } \\
\text { Total }\end{array}$ & $\begin{array}{l}44 \\
19 \\
63\end{array}$ & $\begin{array}{l}29,23 \\
38,42\end{array}$ & 0,057 & $\begin{array}{l}\text { Yes } \\
\text { No } \\
\text { Total }\end{array}$ & $\begin{array}{l}38 \\
25 \\
63\end{array}$ & $\begin{array}{l}26,42 \\
40,48\end{array}$ & 0,002 \\
\hline $\begin{array}{l}\text { Vocational } \\
\text { situation }\end{array}$ & & & & $\begin{array}{l}\text { Vocational } \\
\text { situation }\end{array}$ & & & & $\begin{array}{l}\text { Vocational } \\
\text { situation }\end{array}$ & & & \\
\hline $\begin{array}{l}\text { Yes } \\
\text { No } \\
\text { Total }\end{array}$ & $\begin{array}{l}39 \\
23 \\
62\end{array}$ & $\begin{array}{l}29,29 \\
35,24\end{array}$ & 0,190 & $\begin{array}{l}\text { Yes } \\
\text { No } \\
\text { Total }\end{array}$ & $\begin{array}{l}43 \\
19 \\
62\end{array}$ & $\begin{array}{l}27,97 \\
39,50\end{array}$ & 0,015 & $\begin{array}{l}\text { Yes } \\
\text { No } \\
\text { Total }\end{array}$ & $\begin{array}{l}37 \\
25 \\
62\end{array}$ & $\begin{array}{l}28,16 \\
36,44\end{array}$ & 0,064 \\
\hline $\begin{array}{l}\text { Financial } \\
\text { situation }\end{array}$ & & & & $\begin{array}{l}\text { Financial } \\
\text { situation }\end{array}$ & & & & $\begin{array}{l}\text { Financial } \\
\text { situation }\end{array}$ & & & \\
\hline $\begin{array}{l}\text { Yes } \\
\text { No } \\
\text { Total }\end{array}$ & $\begin{array}{l}39 \\
24 \\
63\end{array}$ & $\begin{array}{l}29,42 \\
36,19\end{array}$ & 0,139 & $\begin{array}{l}\text { Yes } \\
\text { No } \\
\text { Total }\end{array}$ & $\begin{array}{l}44 \\
19 \\
63\end{array}$ & $\begin{array}{l}30,51 \\
35,45\end{array}$ & 0,307 & $\begin{array}{l}\text { Yes } \\
\text { No } \\
\text { Total }\end{array}$ & $\begin{array}{l}38 \\
25 \\
63\end{array}$ & $\begin{array}{l}26,17 \\
40,86\end{array}$ & 0,001 \\
\hline $\begin{array}{l}\text { Leisure } \\
\text { situation }\end{array}$ & & & & $\begin{array}{l}\text { Leisure } \\
\text { situation }\end{array}$ & & & & $\begin{array}{l}\text { Leisure } \\
\text { situation }\end{array}$ & & & \\
\hline $\begin{array}{l}\text { Yes } \\
\text { No } \\
\text { Total }\end{array}$ & $\begin{array}{l}39 \\
24 \\
63\end{array}$ & $\begin{array}{l}31,82 \\
32,29\end{array}$ & 0,919 & $\begin{array}{l}\text { Yes } \\
\text { No } \\
\text { Total }\end{array}$ & $\begin{array}{l}44 \\
19 \\
63\end{array}$ & $\begin{array}{l}30,00 \\
36,63\end{array}$ & 0,175 & $\begin{array}{l}\text { Yes } \\
\text { No } \\
\text { Total }\end{array}$ & $\begin{array}{l}38 \\
25 \\
63\end{array}$ & $\begin{array}{l}28,17 \\
37,82\end{array}$ & 0,036 \\
\hline $\begin{array}{l}\text { Contact with } \\
\text { friends }\end{array}$ & & & & $\begin{array}{l}\text { Contact } \\
\text { with friends }\end{array}$ & & & & $\begin{array}{l}\text { Contact with } \\
\text { friends }\end{array}$ & & & \\
\hline $\begin{array}{l}\text { Yes } \\
\text { No } \\
\text { Total }\end{array}$ & $\begin{array}{l}38 \\
24 \\
62\end{array}$ & $\begin{array}{l}33,92 \\
27,67\end{array}$ & 0,164 & $\begin{array}{l}\text { Yes } \\
\text { No } \\
\text { Total }\end{array}$ & $\begin{array}{l}44 \\
18 \\
62\end{array}$ & $\begin{array}{l}30,70 \\
33,44\end{array}$ & 0,570 & $\begin{array}{l}\text { Yes } \\
\text { No } \\
\text { Total }\end{array}$ & $\begin{array}{l}37 \\
25 \\
62\end{array}$ & $\begin{array}{l}27,46 \\
37,48\end{array}$ & 0,025 \\
\hline Sexual life & & & & Sexual life & & & & Sexual life & & & \\
\hline
\end{tabular}


American Research Journal of Nursing, Volume 1, Issue 3, 2015

ISSN 2379-2922

\begin{tabular}{|c|c|c|c|c|c|c|c|c|c|c|c|}
\hline $\begin{array}{l}\text { Yes } \\
\text { No } \\
\text { Total }\end{array}$ & $\begin{array}{l}38 \\
22 \\
60\end{array}$ & $\begin{array}{l}31,63 \\
28,55\end{array}$ & 0,502 & $\begin{array}{l}\text { Yes } \\
\text { No } \\
\text { Total }\end{array}$ & $\begin{array}{l}42 \\
18 \\
60\end{array}$ & $\begin{array}{l}30,74 \\
29,94\end{array}$ & 0,869 & $\begin{array}{l}\text { Yes } \\
\text { No } \\
\text { Total }\end{array}$ & $\begin{array}{l}36 \\
24 \\
60\end{array}$ & $\begin{array}{l}29,64 \\
31,79\end{array}$ & 0,634 \\
\hline $\begin{array}{l}\text { Activities of } \\
\text { daily life }\end{array}$ & & & & $\begin{array}{l}\text { Activities } \\
\text { of daily life }\end{array}$ & & & & $\begin{array}{l}\text { Activities of } \\
\text { daily life }\end{array}$ & & & \\
\hline $\begin{array}{l}\text { Yes } \\
\text { No } \\
\text { Total }\end{array}$ & $\begin{array}{l}39 \\
24 \\
63\end{array}$ & $\begin{array}{l}30,87 \\
33,83\end{array}$ & 0,404 & $\begin{array}{l}\text { Yes } \\
\text { No } \\
\text { Total }\end{array}$ & $\begin{array}{l}44 \\
19 \\
63\end{array}$ & $\begin{array}{l}33,47 \\
28,61\end{array}$ & 0,195 & $\begin{array}{l}\text { Yes } \\
\text { No } \\
\text { Total }\end{array}$ & $\begin{array}{l}38 \\
25 \\
63\end{array}$ & $\begin{array}{l}30,70 \\
33,98\end{array}$ & 0,351 \\
\hline Family life & & & & Family life & & & & Family life & & & \\
\hline $\begin{array}{l}\text { Yes } \\
\text { No } \\
\text { Total }\end{array}$ & $\begin{array}{l}37 \\
23 \\
60\end{array}$ & $\begin{array}{l}30,89 \\
29,87\end{array}$ & 0,816 & $\begin{array}{l}\text { Yes } \\
\text { No } \\
\text { Total }\end{array}$ & $\begin{array}{l}41 \\
19 \\
60\end{array}$ & $\begin{array}{l}29,49 \\
32,68\end{array}$ & 0,486 & $\begin{array}{l}\text { Yes } \\
\text { No } \\
\text { Total }\end{array}$ & $\begin{array}{l}35 \\
25 \\
60\end{array}$ & $\begin{array}{l}28,90 \\
32,74\end{array}$ & 0,376 \\
\hline $\begin{array}{l}\text { Partner } \\
\text { relationship }\end{array}$ & & & & $\begin{array}{l}\text { Partner } \\
\text { relationship }\end{array}$ & & & & $\begin{array}{l}\text { Partner } \\
\text { relationship }\end{array}$ & & & \\
\hline $\begin{array}{l}\text { Yes } \\
\text { No } \\
\text { Total }\end{array}$ & $\begin{array}{l}39 \\
23 \\
62\end{array}$ & $\begin{array}{l}33,35 \\
28,37\end{array}$ & 0,273 & $\begin{array}{l}\text { Yes } \\
\text { No } \\
\text { Total }\end{array}$ & $\begin{array}{l}44 \\
18 \\
62\end{array}$ & $\begin{array}{l}30,68 \\
33,50\end{array}$ & 0,560 & $\begin{array}{l}\text { Yes } \\
\text { No } \\
\text { Total }\end{array}$ & $\begin{array}{l}37 \\
25 \\
62\end{array}$ & $\begin{array}{l}30,42 \\
33,10\end{array}$ & 0,549 \\
\hline Somatic health & & & & $\begin{array}{l}\text { Somatic } \\
\text { health }\end{array}$ & & & & Somatic health & & & \\
\hline $\begin{array}{l}\text { Yes } \\
\text { No } \\
\text { Total }\end{array}$ & $\begin{array}{l}39 \\
24 \\
63\end{array}$ & $\begin{array}{l}29,90 \\
35,42\end{array}$ & 0,230 & $\begin{array}{l}\text { Yes } \\
\text { No } \\
\text { Total }\end{array}$ & $\begin{array}{l}44 \\
19 \\
63\end{array}$ & $\begin{array}{l}29,67 \\
37,39\end{array}$ & 0,112 & $\begin{array}{l}\text { Yes } \\
\text { No } \\
\text { Total }\end{array}$ & $\begin{array}{l}38 \\
25 \\
63\end{array}$ & $\begin{array}{l}29,47 \\
35,84\end{array}$ & 0,163 \\
\hline $\begin{array}{l}\text { Psychological } \\
\text { health }\end{array}$ & & & & $\begin{array}{l}\text { Psychologic } \\
\text { al health }\end{array}$ & & & & $\begin{array}{l}\text { Psychological } \\
\text { health }\end{array}$ & & & \\
\hline $\begin{array}{l}\text { Yes } \\
\text { No } \\
\text { Total }\end{array}$ & $\begin{array}{l}39 \\
24 \\
63\end{array}$ & $\begin{array}{l}32,44 \\
31,29\end{array}$ & 0,805 & $\begin{array}{l}\text { Yes } \\
\text { No } \\
\text { Total }\end{array}$ & $\begin{array}{l}44 \\
19 \\
63\end{array}$ & $\begin{array}{l}28,61 \\
39,84\end{array}$ & 0,022 & $\begin{array}{l}\text { Yes } \\
\text { No } \\
\text { Total }\end{array}$ & $\begin{array}{l}38 \\
25 \\
63\end{array}$ & $\begin{array}{l}27,39 \\
39,00\end{array}$ & 0,012 \\
\hline
\end{tabular}

Mann Whitney U test was used for analysis.

\section{DISCUSSION}

The aim of our study was to examine if the included breast cancer patients 'estimated life satisfaction was affected during the endocrine treatment, and if it changed over time, and if there was any difference in life satisfaction between patients who experienced side effects compared to patients who did not. The principal findings are that the study group - even at inclusion - scored their life satisfaction lower than the reference group in most of the domains and that their life satisfaction further deteriorated during treatment. At post-treatment the patients had recovered in most domains and their life satisfaction was scored even higher compared with the reference group in some domains. At inclusion, the study group expressed lower life satisfaction in all domains except the vocational situation, financial situation and contact with friends, compared with the reference group. At post-treatment the patients still expressed lower life satisfaction in the domains sexual life, activities of daily living, partner relationship, somatic health and psychological health.

Our study shows that the study group expressed lower life satisfaction compared with the reference group even before the endocrine treatment started. The result is not surprising and correlates with previous studies that have shown that newly diagnosed patients with breast cancer experience reduced life quality related to pain, physical functioning, and health and vitality soon after diagnosis ${ }^{15}$. Younger patients in particular experience poorer healthrelated quality of life after they have been diagnosed with breast cancer ${ }^{16}$. This is probably a result of the negative experience of receiving a breast cancer diagnosis and the breast surgery that they have recently undergone.

The study group showed a numerical decline in estimated life satisfaction in several domains between inclusion and 12 months later (i.e. during therapy). At the latter measurement, the patients who had undergone chemotherapy had 
probably not yet fully recovered from that treatment and at the same time most of the side effects from the endocrine treatment had probably set in ${ }^{17}$. In a prospective cohort study from 2012, more than $50 \%$ of patients who had recently begun AI treatment had new or worsened musculoskeletal pain and these symptoms generally occurred within 8 weeks of the start of the endocrine treatment ${ }^{17}$. In our study the patients had recovered in most respects 2 years after completed treatment. There were large improvements in scored life satisfaction when comparing inclusion and post-treatment scores, with significant improvement in several domains.

Little is known about how breast cancer patients receiving endocrine treatment recover but some earlier studies have shown that, in general, breast cancer patients recover over time. In an Australian study it was shown that, after 3 years, breast cancer patients had the same level of health-related quality of life as pre-diagnosis in most domains ${ }^{15}$. In Danish, age-stratified random sample of breast cancer patients a comparison of quality of life was made between the general female population and women who had been diagnosed with breast cancer 5 years previously. The quality of life was almost equal: the breast cancer patients reported better general health but worse mental health ${ }^{18}$. In our study there was a distinctly lower score for the domain sexual life both at inclusion and during treatment compared with the reference group and it is notable that this persisted at the post-treatment measurement.

During the treatment significant differences were seen in how patients with reported symptoms estimated their life satisfaction compared with those without reported symptoms. In our study it was shown that the patients with symptoms estimated lower life satisfaction in most domains. In another study it was reported that the side effects of the endocrine treatment could be so severe that they even complicated the patients' return to work ${ }^{19}$.

The most substantial advantage of the present study is its long term follow-up, i.e. the patients have also been followed after the completion of endocrine. Other methodological advantages are the prospective design with a consecutive clinical follow-up and the use of a familiar and validity-tested questionnaire. A limitation is, however, the patient characteristics. The study group may not be representative of all breast cancer patients since it consisted of patients that were active professionally, the majority had at least upper secondary school education and all of the patients could read and understand Swedish. Another limitation is the small sample size, which may have resulted in failure to detect associations between estimated life satisfaction and the other factors studied.

\section{CONCLUSION}

In this study the important findings are that the studied breast cancer patients receiving endocrine treatment estimated their life satisfaction lower in most domains compared with the normal population already at inclusion. During the treatment, life satisfaction further deteriorated but the patients had recovered in most domains post-treatment. Notable is the deterioration in sexual life and partner relationship at post-treatment compared with inclusion. This study indicates that long-term effects of endocrine breast cancer therapy need to be further researched, with a particular focus on issues relating to sexuality and relationships.

\section{ACKNOWLEDGEMENT}

The study was financed by the Breast Cancer Foundation in Sweden and Brunetti-Jacovone Foundation.

\section{Statement of Competing Interests}

There are no competing interests.

\section{REFERENCES}

[1] Socialstyrelsen. Cancer incidence in Sweden 2013. Stockholm: Socialstyrelsen; 2014.

[2] Davies C, Pan H, Godwin J, Gray R, Arriagada R, Raina V, et al. Long-term effects of continuing adjuvant tamoxifen to 10 years versus stopping at 5 years after diagnosis of oestrogen receptor-positive breast cancer: ATLAS, a randomised trial. Lancet. 2013; 381(9869): 805-16.

[3] Burstein HJ, Prestrud AA, Seidenfeld J, Anderson H, Buchholz TA, Davidson NE, et al. American Society of Clinical Oncology clinical practice guideline: update on adjuvant endocrine therapy for women with hormone receptor-positive breast cancer. Journal of clinical oncology : official journal of the American Society of Clinical Oncology. 2010; 28(23): 3784-96.

[4] McCowan C, Wang S, Thompson AM, Makubate B, Petrie DJ. The value of high adherence to tamoxifen in women with breast cancer: a community-based cohort study. British journal of cancer. 2013; 109(5): 1172-80. 
[5] Wigertz A, Ahlgren J, Holmqvist M, Fornander T, Adolfsson J, Lindman H, et al. Adherence and discontinuation of adjuvant hormonal therapy in breast cancer patients: a population-based study. Breast cancer research and treatment. 2012; 133(1): 367-73.

[6] Buijs C, de Vries EG, Mourits MJ, Willemse PH. The influence of endocrine treatments for breast cancer on health-related quality of life. Cancer Treat Rev. 2008; 34(7): 640-55.

[7] Mok K, Juraskova I, Friedlander M. The impact of aromatase inhibitors on sexual functioning: current knowledge and future research directions. Breast. 2008; 17(5): 436-40.

[8] Din OS, Dodwell D, Wakefield RJ, Coleman RE. Aromatase inhibitor-induced arthralgia in early breast cancer: what do we know and how can we find out more? Breast cancer research and treatment. 2010; 120(3): 525-38.

[9] Burstein HJ, Winer EP. Aromatase inhibitors and arthralgias: a new frontier in symptom management for breast cancer survivors. Journal of clinical oncology : official journal of the American Society of Clinical Oncology. 2007; 25(25): 3797-9.

[10] Coleman RE, Bolten WW, Lansdown M, Dale S, Jackisch C, Merkel D, et al. Aromatase inhibitor-induced arthralgia: clinical experience and treatment recommendations. Cancer Treat Rev. 2008; 34(3): 275-82.

[11] Henry NL, Azzouz F, Desta Z, Li L, Nguyen AT, Lemler S, et al. Predictors of aromatase inhibitor discontinuation as a result of treatment-emergent symptoms in early-stage breast cancer. Journal of clinical oncology : official journal of the American Society of Clinical Oncology. 2012; 30(9): 936-42.

[12] Huang X, Zhang Q, Kang X, Song Y, Zhao W. Factors associated with cancer-related fatigue in breast cancer patients undergoing endocrine therapy in an urban setting: a cross-sectional study. BMC Cancer. 2010; 10: 453.

[13] Navari RM, Brenner MC, Wilson MN. Treatment of depressive symptoms in patients with early stage breast cancer undergoing adjuvant therapy. Breast cancer research and treatment. 2008; 112(1): 197-201.

[14] Fugl-Meyer AR, Melin R, Fugl-Meyer KS. Life satisfaction in 18- to 64-year-old Swedes: in relation to gender, age, partner and immigrant status. J Rehabil Med. 2002; 34(5): 239-46.

[15] Leung J, Pachana NA, McLaughlin D. Social support and health-related quality of life in women with breast cancer: a longitudinal study. Psycho-oncology. 2014; 23(9): 1014-20.

[16] Hoyer M, Johansson B, Nordin K, Bergkvist L, Ahlgren J, Lidin-Lindqvist A, et al. Health-related quality of life among women with breast cancer - a population-based study. Acta oncologica. 2011; 50(7): 1015-26.

[17] Singer O, Cigler T, Moore AB, Levine AB, Hentel K, Belfi L, et al. Defining the aromatase inhibitor musculoskeletal syndrome: a prospective study. Arthritis Care Res (Hoboken). 2012; 64(12): 1910-8.

[18] Peuckmann V, Ekholm O, Rasmussen NK, Moller S, Groenvold M, Christiansen P, et al. Health-related quality of life in long-term breast cancer survivors: nationwide survey in Denmark. Breast cancer research and treatment. 2007; 104(1): 39-46.

[19] Johnsson A, Fornander T, Olsson M, Nystedt M, Johansson H, Rutqvist LE. Factors associated with return to work after breast cancer treatment. Acta Oncologica. 2007; 46(1): 90-6. 